\title{
Health behaviour models: a framework for studying adherence in children with atopic dermatitis
}

\author{
S. S. Chisolm,* S. L. Taylor,* J. G. Gryzwacz,† J. L. O’Neill,* R. R. Balkrishnan‡ and S. R. Feldman*§ণ \\ Departments of *Dermatology and †Family Medicine, Wake Forest University School of Medicine, Winston, Salem, NC, USA; ¥Schools of Pharmacy and Public \\ Health, University of Michigan, Ann Arbor, MI, USA; Departments of §Pathology and $\uparrow$ Public Health Sciences, Wake Forest University School of Medicine, \\ Winston, Salem, NC, USA \\ doi:10.1111/j.1365-2230.2009.03741.x
}

\section{Summary}

\begin{abstract}
Atopic dermatitis $(\mathrm{AD})$ is a common problem of childhood causing considerable distress. Effective topical treatments exist, yet poor adherence often results in poor outcomes. A framework is needed to better understand adherence behaviour. To provide a basis for this framework, we reviewed established models used to describe health behaviour. Structural elements of these models informed the development of an adherence model for AD that can be used to complement empirical AD treatment trials. Health behaviour models provide a means to describe factors that affect adherence and that can mediate the effects of different adherence interventions. Models of adherence behaviour are important for promoting better treatment outcomes for children with $\mathrm{AD}$ and their families. These models provide a means to identify new targets to improve adherence and a guide for refining adherence interventions.
\end{abstract}

\section{Introduction}

Atopic dermatitis (AD) is an important and increasing healthcare problem in children. ${ }^{1-3} \mathrm{AD}$ can cause discomfort, loss of sleep, fatigue and psychosocial distress. ${ }^{3}$ Parents and caregivers can also experience significant psychosocial distress and fatigue. ${ }^{3}$ Loss of productivity in school and at work adds to the burden of the disease.

There are many effective treatments for AD. Topical corticosteroids and other immunomodulators are highly effective in clinical trials. ${ }^{4}$ Despite this, AD remains poorly controlled in many patients, resulting in poor quality of life for both patients and their caregivers. Failure of topical treatments to achieve success may also lead to use of potentially toxic systemic treatments.

Correspondence: Dr Steven R. Feldman, Department of Dermatology, Wake Forest University School of Medicine, Medical Center Boulevard, WinstonSalem, NC 27157-1071, USA

E-mail: sfeldman@wfubmc.edu

Conflict of interest: The Center for Dermatology Research is supported by an educational grant from Galderma Laboratories, L.P.

Accepted for publication 20 August 2009
'Adherence', a term that has replaced the more pejorative 'compliance' concept, probably plays a major role in $\mathrm{AD}$ treatment failures.

Poor adherence is common and has a tremendous effect on health and healthcare expenditures. ${ }^{5-7}$ Adherence can be poor even when a simple, static treatment regimen is used. ${ }^{8}$ In one study of children with $\mathrm{AD}$, adherence to topical triamcinolone was assessed with electronic monitors; the mean adherence rate was only $32 \%$ over an 8 -week period. ${ }^{8}$ Even patients who have objectively and subjectively severe atopic skin disease may not be using their recommended treatment. ${ }^{9}$ Adherence rates are likely to be even lower over the extended course of this chronic disease and when dynamic, multi-agent regimens are prescribed. Poor adherence has the potential to cause both initial treatment failure and later loss of treatment effectiveness (so-called 'tachyphylaxis'). ${ }^{8}$ Models of AD treatment that do not account for adherence may be inadequate for describing the relationship between treatment and outcomes seen in clinical practice (Fig. 1).

The purpose of this paper is to review existing models of health behaviour and to use elements of those models 


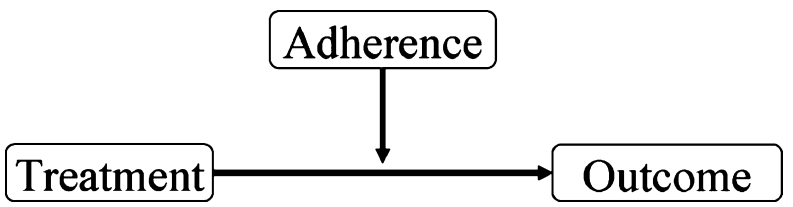

Figure 1 Patient adherence to treatment affects disease outcome. Atopic dermatitis (AD) treatment studies are often based on a very simple model: that treatment of the disease improves the outcome. Such models do not account for the rich variation in human behaviour that contributes to the outcome of treatment. The effect of any treatment on a disease outcome is contingent upon adherence to the recommended treatment. Adherence is a measurable variable, and accounting for adherence improves understanding of the relationship between a treatment and its effect on disease outcome. The development of interventions that improve adherence may be just as valuable as new pharmacological agents in improving treatment outcomes in AD. Similarly, a better understanding of factors that affect adherence is as important to the development of adherence interventions as an understanding of immune mechanisms in $\mathrm{AD}$ is to the development of new pharmacological therapies.

to describe a general model that can be used to better understand, study and manipulate $\mathrm{AD}$ adherence behaviour.

\section{Existing models of health behaviour}

Adherence behaviour management plays a substantial role in treatment regimens of diabetes, hypertension, chronic heart failure and asthma. Models have been developed to incorporate the factors affecting adherence into health recommendations. These models provide a basis for modelling $\mathrm{AD}$ adherence behaviour.

There are three major models used to describe patients' health behaviours: the Health Belief Model, the Health Behaviour Model, and the Social Cognitive Theory. The Health Belief Model is based on the concept that people make rational choices about whether to engage in an advocated behaviour. ${ }^{10-14}$ In essence, people are believed to engage in a rational assessment of the known or presumed costs associated with an advocated behaviour relative to the anticipated benefits. The Health Belief Model includes four main concepts that shape individual assessment and appraisal of potential costs and benefits: (i) a patient's perceived susceptibility to a particular condition; (ii) perceived severity if the patient were to develop the condition, including the combined effect of disease, treatment and sequelae on overall quality of life; (iii) perceived benefits (a patient's judgement of how much a recommended action will reduce their risk of developing the condition or mitigate its negative consequences); and (iv) perceived barriers (a patient's judgement of the aggregate costs of the recommended action, including financial, psychological and time costs to the patient or their family). More recent forms of the Health Belief Model further outline two other factors in the decision to engage in a behaviour: (i) self-efficacy, which is the patient's belief that they are capable of taking the recommended action; and (ii) cues to action, which are aids that teach or remind the patient about the recommended action. ${ }^{10}$

Although the Health Belief Model identifies internal processes shaping health behaviours, the Health Behaviour Model highlights external factors that either shape patients' thinking or exert direct effects on behaviour. Modifications to the Health Behaviour Model, which led to the Health Care Delivery Model devised by Andersen and Aday, ${ }^{15}$ emphasize the effect of external enabling factors on patient adherence. These external factors include both contextual barriers in patients' and caregivers' lives, and characteristics of the healthcare system. External factors, particularly changes over which the clinician has control and that are feasible at the practice level, may be especially good targets for adherence interventions (Fig. 2).

The Social Cognitive Theory emphasizes the concept of reciprocal determinism (a 'continuous, dynamic interaction between the individual, the environment and behaviour'). ${ }^{10,14}$ This is an important consideration in adherence behaviour because outcomes of treatment may affect subsequent adherence behaviour (Fig. 3). In particular, better initial treatment outcomes may

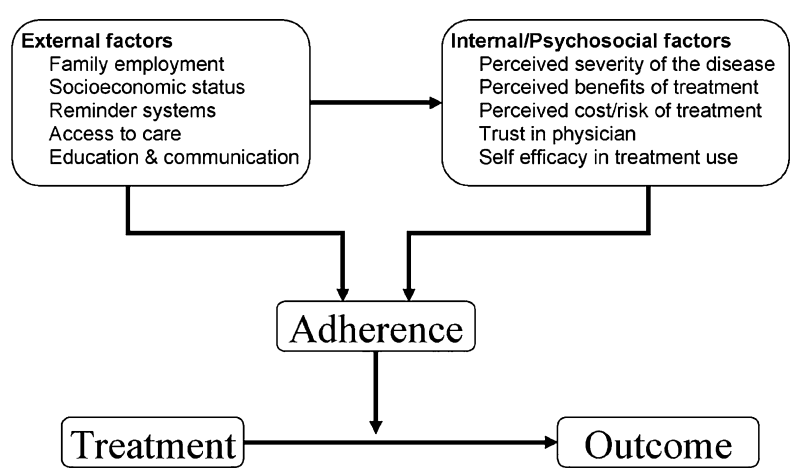

Figure 2 Internal and external factors that affect adherence behaviour. Adherence behaviour is shaped by both internal and external factors. The external factors may have direct effects on adherence or may modulate patients' internal thinking. External factors are good targets for adherence interventions and include both contextual barriers in the lives of patients/caregivers and characteristics of the healthcare system. 


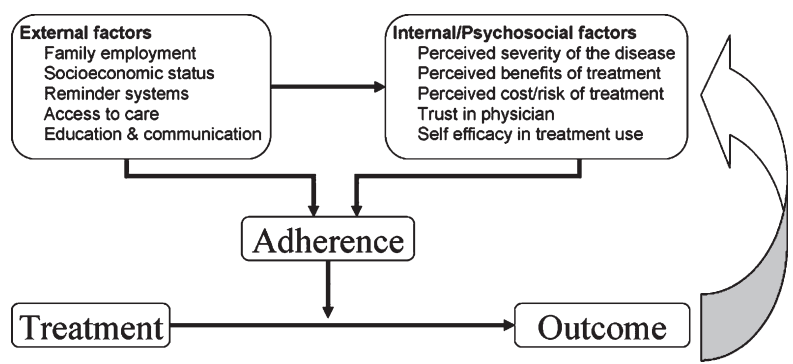

Figure 3 Outcomes of treatment may affect adherence behaviour. There is a reciprocal relationship between treatment outcomes and adherence behaviour. Better treatment outcomes may positively affect internal factors that guide patients' adherence behaviour. Recognizing this relationship helps identify the potential to use programmes that improve initial treatment outcomes to help further promote better adherence.

increase the perceived value of a treatment, resulting in better adherence.

\section{Integrating existing models to form an adherence model for $A D$}

Based on the existing models, internal/psychosocial factors that are anticipated to modulate patients' adherence include perceptions about the severity of disease; the benefits, risks and costs of treatment; trust in the clinician; and self-efficacy in treatment use (Fig. 3). External factors that can be manipulated include reminder systems, access to care, and patient education (Fig. 3). Modelling predicts that clinicians can leverage several factors to promote better adherence including increasing trust in the clinician, providing specific cues to action and reducing the perceived burden of treatment. The effect of such interventions can be tested in clinical trials using objective monitoring of adherence behaviour.

A critical feature of model-based research is the ability to test and examine the mechanisms by which interventions improve outcomes. AD treatment education programmes can improve adherence, but as yet we do not know why. ${ }^{16-18}$ The educational programme could work simply by reducing patients' fears, or it may be effective because it elicits greater trust in the clinician, more support from family members, incorporation of cues to action, or a reduction in the apparent burden of treatment. By knowing specifically how educational interventions improve AD adherence, newer, less complicated and perhaps even more effective interventions could be developed.

Written action plans (WAPs), widely used in treating children with asthma, provide a potential intervention to improve adherence in children with AD. ${ }^{19}$ WAPs are designed to empower patients to respond appropriately to the waxing and waning course of the disease. WAPs target factors that are central to adherent behaviour: cues to action (discrete criteria that patients can use to easily determine if action is required); self-efficacy over the health condition; and a sense of treatment efficacy. Both the content of the WAP and the communication between patient and provider when the WAP is developed are intended to convey the message, 'this plan can help you control your condition'. Asthma and $\mathrm{AD}$ share a chronic relapsing and remitting course that requires variation in management over time, making AD another logical target for a WAP intervention. ${ }^{19}$ Development and testing of a WAP for AD would focus on whether the effect of the WAP on adherence is a function of improved trust, improved self-efficacy, improved perceived risk-benefit ratio, or some combination of these effects (Fig. 4).

Follow-up consultations increase patients' use of medication, and the use of an early follow-up visit has also been proposed as a means to improve adherence in patients with $\mathrm{AD}{ }^{8,20}$ This intervention is anticipated to function differently from a WAP. An early follow-up intervention increases patient and parent willingness to adhere to treatment over the short term. ${ }^{21}$ The improved adherence to treatment that occurs for a short time before a clinic visit is known as 'white-coat compliance'. $8,22,23$ The feedback of outcome on adherence in the adherence behaviour model (a combination of factors in the three models outlined above) (Fig. 3) predicts that an early return visit intervention may also improve long-term $\mathrm{AD}$ treatment outcomes by

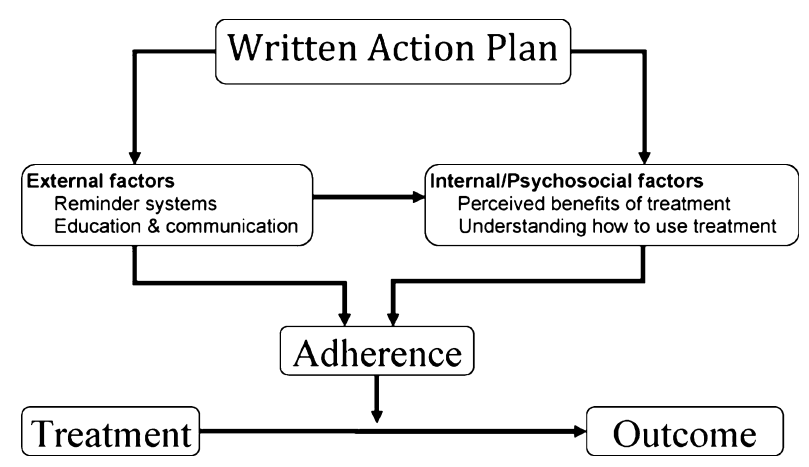

Figure 4 Modelling the mechanism for the effects of a written action plan (WAP) on adherence behaviour. A model provides a basis for determining how an intervention functions. The model of a WAP intervention predicts several mechanisms by which a WAP can enhance adherence behaviour. Measuring these factors in a WAP intervention trial allows identification of how the WAP is functioning. This may help facilitate refinement of the intervention to achieve improved adherence. 
increasing patients' and caregivers' perceptions of treatment efficacy and thus increasing their ongoing level of adherence. Analysis of an early follow-up intervention would help refine the model of adherence behaviour, determining not only whether the intervention is effective, but also whether it is acting as expected on other points within the model.

\section{Conclusion}

Scientific models are used to systematically guide research, to understand the most salient leverage points for intervention, and to guide study design and evaluation. In studies of the immunology of $\mathrm{AD}$, researchers develop comprehensive models of cells, adherence proteins and cytokines to guide hypothesis-based research. Likewise, in the realm of behavioural research, a comprehensive conceptual model is needed. Conceptual models help guide the design of empirical studies, the interpretation of resultant findings, the development of clinical intervention trials, and, ultimately, the practice of evidence-based medicine and clinical care.

Consider a child with $\mathrm{AD}$ who is not improving with treatment as expected. We know that poor adherence to treatment is a likely cause, as simply admitting the patient to the hospital and applying the medication on an inpatient basis typically results in very rapid clearing of the disease. There could be many reasons for nonadherence. The parents may not have the financial or time resources to purchase or use the medication. The parents may be fearful of the potential side-effects of treatment, or they may not trust that the medication will work. They may not understand how the medication is supposed to be used. They may simply forget to apply the medication, or the child might refuse to allow the medicine to be applied because they do not like how it feels. A model that organizes and describes the factors affecting adherence and the resulting effect on outcomes provides a framework for caring for patients and for developing and refining targeted interventions.

The outcomes of the most prevalent skin diseases are inextricably linked with human behaviours. Although human behaviour is complex, it is perhaps no more complex than well-studied pathophysiological systems. A basic understanding of the factors that influence health behaviours and how healthcare interactions can be optimized to prevent or manage these diseases is vital to the advancement of skin disease treatment. Human behaviour must be assessed with the same scientific rigour as other disciplines of basic investigational dermatology. Our nascent collective understanding of adherence behaviour will benefit from models that frame the adherence problem. These models will provide a rational framework for hypothesis-testing research and for further theoretical development. It is hoped that these models will form a framework for future research that will benefit both paediatric patients with $\mathrm{AD}$ and those with other dermatological diseases.

\section{Learning points}

- Atopic dermatitis (AD) is an important disease of childhood that causes psychosocial distress for both patients and caregivers. Nonadherence to topical medications probably plays a role in $\mathrm{AD}$ treatment failures.

- Scientific models are used to systematically guide research and study design, to interpret results and develop appropriate interventions, and to enhance the practice of evidence-based medicine.

- Conceptual models are needed for the treatment of AD. Failure to account for the role of decreased adherence in $\mathrm{AD}$ treatment failures may result in inadequate models.

- The three major models to describe health behaviours are: the Health Belief Model, the Health Behaviour Model and the Social Cognitive Theory. Existing models can be used to develop an adherence model for AD.

- Modelling predicts that clinicians can leverage several factors to improve adherence, e.g. increasing trust in clinicians, providing specific treatment cues and decreasing the burden of treatment. The effect of these interventions can be tested in studies using objective adherence monitoring.

- Understanding the mechanism by which proposed interventions improve adherence is essential to refining models of treatment adherence.

\section{References}

1 Schafer T, Ring J. Epidemiology of allergic diseases. Allergy 1997; 52: 14-22.

2 Schultz LF. Atopic dermatitis: an increasing problem. Pediatr Allergy Immunol 1996; 7: 51-3.

3 Carroll CL, Balkrishnan R, Feldman SR et al. The burden of atopic dermatitis: impact on the patient, family, and society. Pediatr Dermatol 2005; 22: 192-9.

4 Krakowski AC, Eichenfield LF, Dohil MA. Management of atopic dermatitis in the pediatric population. Pediatrics 2008; 122: 812-24. 
5 Richards HL, Fortune DG, O'Sullivan TM et al. Patients with psoriasis and their compliance with medication. J Am Acad Dermatol 1999; 41: 581-3.

6 Meredith PA. Therapeutic implications of drug 'holidays'. Eur Heart J 1996; 17: 21-4.

7 Berg JS, Dischler J, Wagner DJ et al. Medication compliance: a healthcare problem. Ann Pharmacother 1993; 27: S1-24.

8 Feldman SR, Camacho FT, Krejci-Manwaring J et al. Adherence to topical therapy increases around the time of office visits. J Am Acad Dermatol 2007; 57: 81-3.

9 Brown KL, Krejci-Manwaring J, Tusa MG et al. Poor compliance with topical corticosteroids for atopic dermatitis despite severe disease. Dermatol Online J 2008; 14: 13.

10 Redding CA, Rossi JS, Rossi SR et al. Health Behavior Models. Available at: http://drzaius.ics.uci.edu/meta/ classes/informatics161_fall06/papers/10a-Redding HealthBehaviorModels.pdf. (accessed 11 November 2008).

11 Becker MH, Drachman RH, Kirscht JP. A new approach to explaining sick-role behavior in low-income populations. Am J Public Health 1974; 64: 205-16.

12 Becker MH, Maiman LA. Sociobehavioral determinants of compliance with health and medical care recommendations. Med Care 1975; 13: 10-24.

13 Janz NK, Becker MH. The Health Belief Model: a decade later. Health Educ Q 1984; 11: 1-47.

14 Bandura A. Social Foundations of Thought and Action: A Social Cognitive Theory. Englewood Cliffs: NJ Prentice Hall, 1986.

15 Aday LA, Andersen R. A framework for the study of access to medical care. Health Serv Res 1974; 9: 208-20.
16 Chida Y, Steptoe A, Hirakawa N et al. The effects of psychological intervention on atopic dermatitis. A systematic review and meta-analysis. Int Arch Allergy Immunol 2007; 144: 1-9.

17 Weber MB, Fontes Neto PT, Prati C et al. Improvement of pruritus and quality of life of children with atopic dermatitis and their families after joining support groups. J Eur Acad Dermatol Venereol 2008; 22: 992-7.

18 Staab D, von Rueden U, Kehrt R et al. Evaluation of a parental training program for the management of childhood atopic dermatitis. Pediatr Allergy Immunol 2002; 13: 84-90.

19 Chisolm SS, Taylor SL, Balkrishnan R, Feldman SR. Written action plans: potential for improving outcomes in children with atopic dermatitis. J Am Acad Dermatol 2008; 59: $677-83$.

20 Krejci-Manwaring J, Tusa MG, Carroll C et al. Stealth monitoring of adherence to topical medication: adherence is very poor in children with atopic dermatitis. J Am Acad Dermatol 2007; 56: 211-16.

21 Yentzer BA, Camacho FT, Young T et al. Good adherence and early efficacy using desonide hydrogel for atopic dermatitis: results from a program addressing patient compliance. J Drugs Dermatol 2009 (in press).

22 Cramer JA, Scheyer RD, Mattson RH. Compliance declines between clinic visits. Arch Intern Med 1990; 150: 1509-10.

23 Smith DM, Norton JA, Weinberger M et al. Increasing prescribed office visits. A controlled trial in patients with diabetes mellitus. Med Care 1986; 24: 189-99. 